\title{
Tests on a mock-up of the feedback controlled matching options of the ITER ICRH system
}

\author{
D. Grine, M. Vervier, A. Messiaen, P. Dumortier \\ Lab. for Plasma Physics - Royal Military Academy, 30 avn. de la Rennaissance, Brussels, Belgium
}

\begin{abstract}
Automatic control of the matching of the ITER ICRH antenna array on a reference load is presently developed and tested for optimization on a low-powered scaled (1:5) mock-up. Resilience to fast load variations is obtained either by 4 Conjugate-T (CT) or 4 quadrature hybrid circuits, the latter being the reference option. The main results are (i) for the CT option: successful implementation of the simultaneous feedback control of 11 actuators for the matching of the 4 CT and for the control of the array toroidal phasing; (ii) for the hybrid option: the matching and the array current control via feedback control of the decouplers and double stub tuners. This system is being progressively implemented and the simultaneous control of matching and antenna current has already been successfully tested on half of the array for heating and current drive phasings.
\end{abstract}

Keywords: Load-resilience, Conjugate-T, hybrid, antenna, matching, ITER, ICRF

\section{INTRODUCTION}

For the ICRF heating or current drive of ITER, 20MW must be coupled to the plasma in the $40-55 \mathrm{MHz}$ frequency band via a tight array of 24 radiating shorted straps fed by four power lines. The straps are grouped in 8 triplets and connected to 4-port junctions. The matching system must be resilient $(|\Gamma|<0.2)$ for a wide range of fast antenna load variations - with respect to the reference load - due to the ELMs. In addition, the matching system must include control of the currents in the straps to impose the radiation spectrum of the array for plasma heating or current drive. The proposed matching systems alleviate the adverse effects of strong mutual coupling between the matching circuits and power sources by the use of appropriate decoupler circuits. The two options considered for ITER are examined: the circuit using four $3 \mathrm{~dB}$ hybrids, which is the reference option, and the one using four Conjugate-T matching circuits, which is the back-up option [1]. The automatic matching systems are tested on a mock-up (scale 1:5) of the antenna plug facing a movable waterload at a frequency of the ITER band multiplied by the inverse of the scale factor.

\section{MATCHING SYSTEM AND ALGORITHMS}

\section{Conjugate-T}

Figure 1 shows the layout of the antenna plug (mock-up) followed by the 8 toroidal decouplers, the 4 CT circuits and the 4 second matching stages. Preset are: (i) the locations of the 6 toroidal decouplers at voltage anti-node $(\mathrm{A}-\mathrm{H})$, (ii) the central impedance 
of each decoupler to minimize the mutual coupling between adjacent matching circuits, (iii) the second stages to set the best impedance $Z_{C T}$ at the output of the CT's for loadresilience (from modeling) and (iv) the forward power of the 4 sources to set the relative voltage amplitudes in A-H (also from modeling). Two feedback loops are acting simultaneously on the system: (i) the matching feedback on the two tuning stubs of each CT $(\mathrm{A}-\mathrm{B}, \mathrm{C}-\mathrm{D}, \ldots)$ to bring below a chosen threshold the reflection coefficient of each generator and (ii) the phase feedback acting on the generator's relative phase to control the phase of the voltages at the ports $\mathrm{A}-\mathrm{H}$ in order to control the strap current phasing.

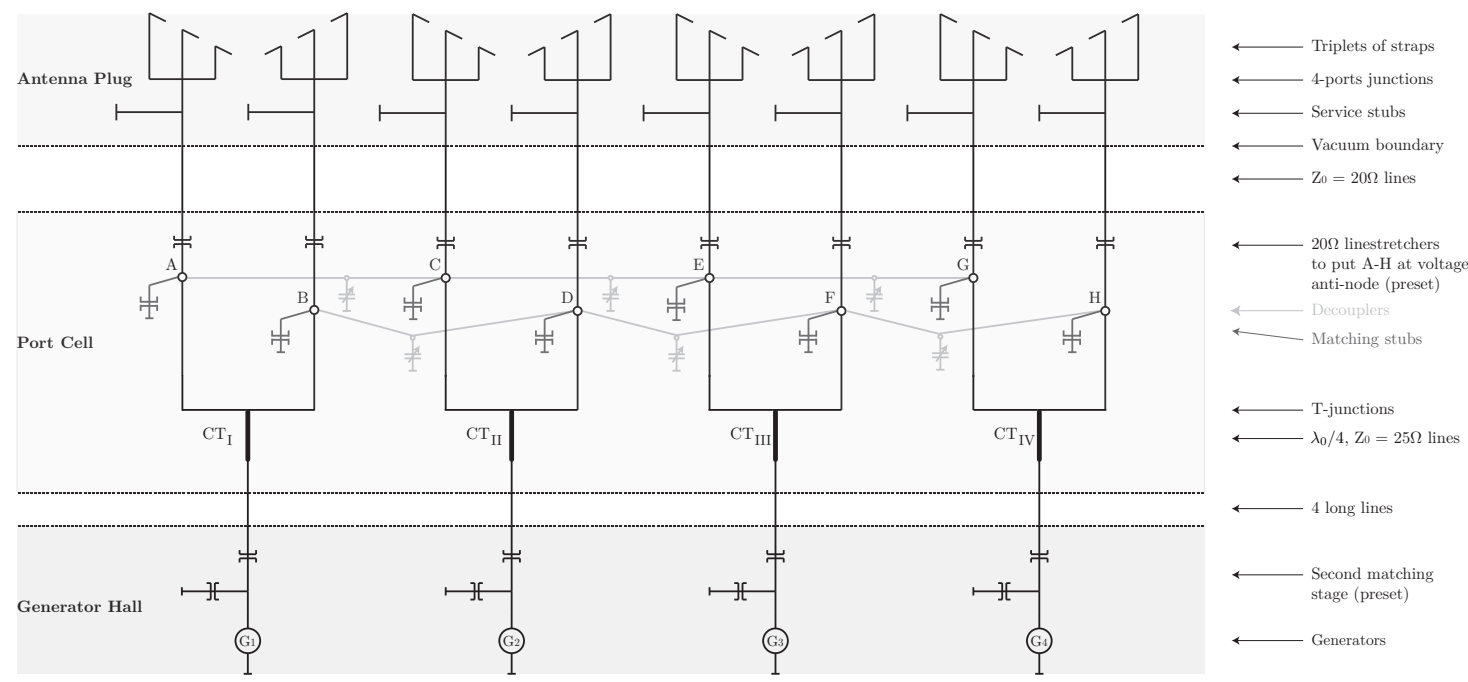

FIGURE 1. Conjugate-T matching circuit (mock-up)

The matching algorithm for the feedback is straightforward: for each CT the 2 stubs are displaced one after the other, if the magnitude of the reflection coefficient at their corresponding power source increases, the direction of their displacement is reversed (see the principle on Figure 2, left-side). Full details are given in [2].
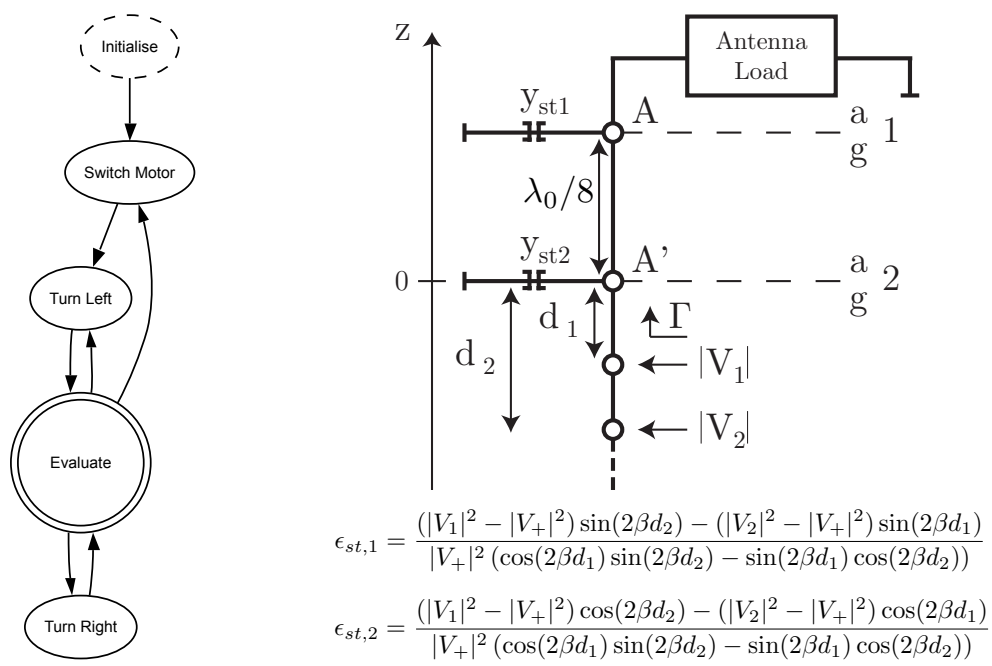

FIGURE 2. Matching algorithms: a). Conjugate-T (left-side) and b). hybrid (right-side) 


\section{3dB Hybrid}

Figure 3 shows the layout of the hybrid matching circuit: four $3 \mathrm{~dB}$ hybrids are connected to lines from adjacent poloidal triplets $(\Delta \Phi=\pi / 2)$ and fed by four power sources with preset phase and same forward power. The locations $\mathrm{A}-\mathrm{H}$ are also preset at voltage anti-node by $20 \Omega$ line stretchers. Various simultaneous feedbacks are active: (i) feedback on the double stub tuners (A-H and $\left.\mathrm{A}^{\prime}-\mathrm{H}^{\prime}\right)$ to minimize the reflection coefficient amplitude at the outputs of each hybrid and (ii) feedback on the poloidal decouplers and either the toroidal ones (case of current drive phasing) or the toroidalpoloidal ones (case of heating phasing) to control the amplitude at the voltage anti-node points A-H while maintaning the mutual coupling at a low level [1] [3].

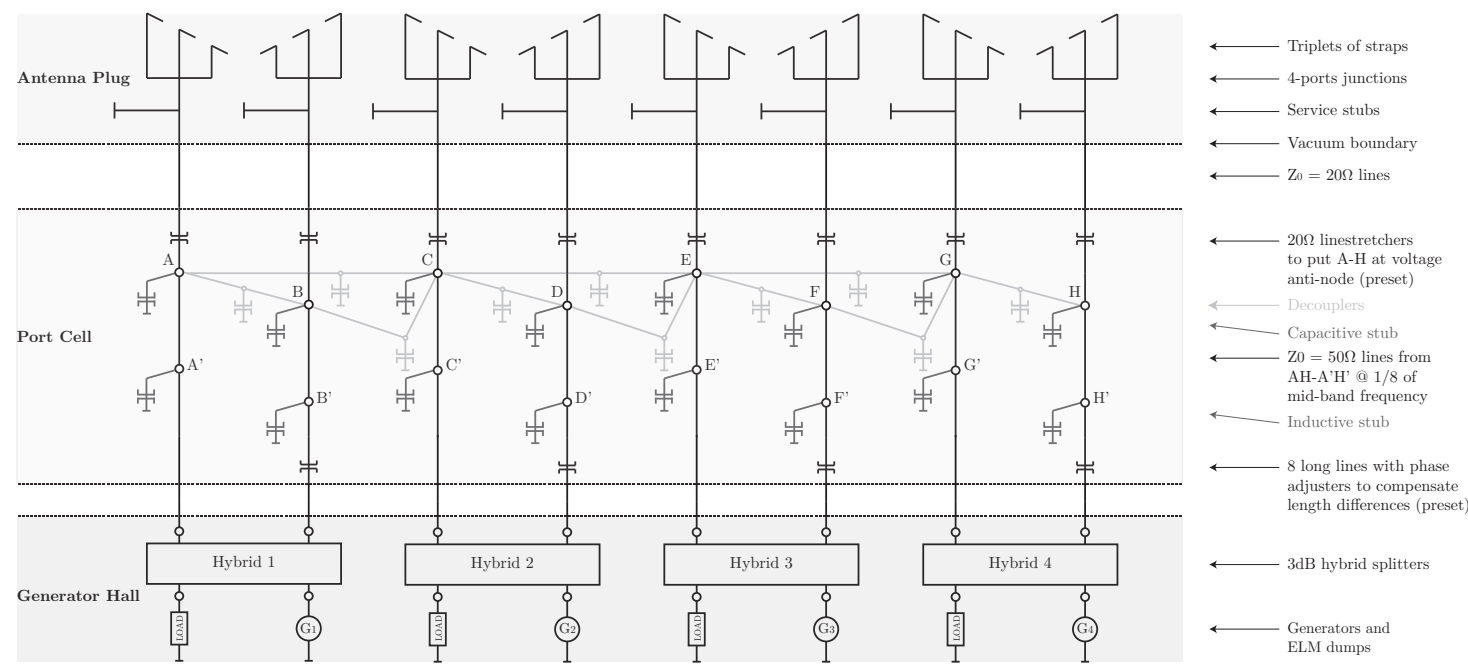

FIGURE 3. $3 \mathrm{~dB}$ hybrid matching circuit (mock-up)

The matching algorithm uses $\Gamma$ on each line: the first stub alters the real part of $\Gamma$ while the second - at an eighth of the electrical wavelength $\lambda_{0}$ (mid-band frequency) further - alters the imaginary part. To avoid phase measurements, error signals are derived that solely use voltage magnitudes. The resulting algorithm is displayed on Figure 2 (rightside) and shows the voltage magnitude probes at a distance $d_{1}$ and $d_{2}$ respectively of the second junction ( $\left.\mathrm{A}^{\prime}-\mathrm{H}^{\prime}\right)$ as well as expressions for $\varepsilon_{S t_{1}}$ and $\varepsilon_{S t_{2}}$.

Feedback on the decouplers to place points $\mathrm{A}-\mathrm{H}$ at equal voltage amplitude uses measurements of the voltage magnitude at the 8 input ports of the circuit and the magnitude difference is used as error signal as shown on Figure 4 (left-side). Due to the phase difference between the two ports of a decoupler: 23 of the 26 actuators are feedback controlled at a time (depending on the type of heating or current drive), the others being preset. All tunable stubs use bang-bang control and position and velocity control of the line stretchers is achieved via Pulse Width Modulation.

Finally, the circuit is being progressively implemented and already half of the system has been succesfully tested. For example, Figure 4 (right-side) shows voltage magnitudes (A-D as indicated on Figure 3) converging after an abrupt change in antenna load. 

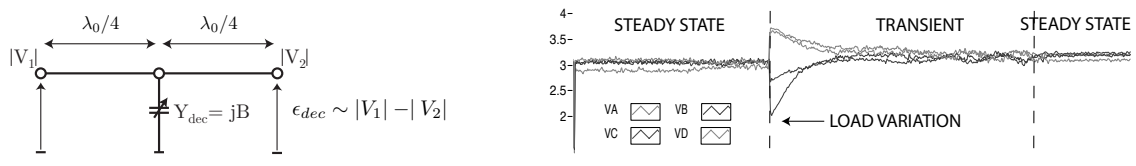

FIGURE 4. Hybrid decoupler: schematic (left) mock-up measurement (right)

\section{RESULTS}

Load-resilience for $\mathrm{R}_{\mathrm{a}, \text { eff }} \approx 2 \Omega / \mathrm{m}$ and current drive $(0, \pi / 2, \pi, 3 \pi / 2)$ is shown in Figure 5. Similar results have been obtained for various heating scenarios: (i) $0,0, \pi, \pi$; $0, \pi, \pi, 0 ; 0, \pi, 0, \pi$ for the CT circuit and (ii) 0,$0 ; 0, \pi$ for the hybrid circuit. In the case of the hybrid circuit, simultaneous control of 12 actuators has already been achieved.
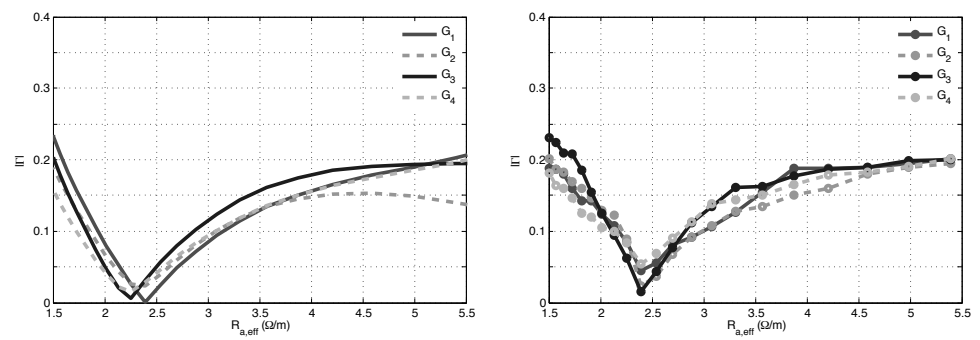

FIGURE 5. Load-resilience Conjugate-T circuit: simulation (left) and mock-up measurements (right)
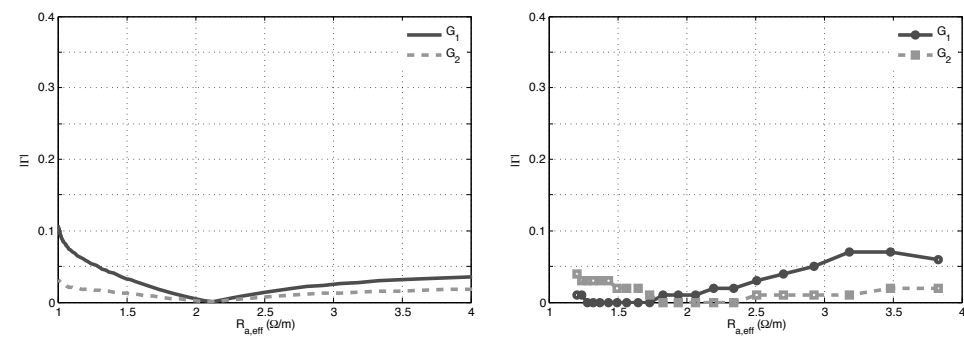

FIGURE 6. Load-resilience $3 \mathrm{~dB}$ hybrid circuit: simulation (left) and mock-up measurements (right)

Finally, good correlation between simulations and measurements is observed as well as superior performance of the hybrid matching circuit.

This work, supported by the European Communities under the contract of Association EURATOM/Belgian state, was carried out within the framework of the European Fusion Development Agreement. The views and opinions expressed herein do not necessarily reflect those of the European Commission.

\section{REFERENCES}

1. A. Messiaen, M. Vervier, P. Dumortier, D. Grine, P. Lamalle, F. Durodié, R. Koch, F. Louche, and R. Weynants, Nuclear Fusion 49 (2009).

2. D. Grine, Master Thesis Ghent University; LPP-ERM/KMS lab. Report no 134 (2008).

3. M. Vervier, A. Messiaen, and P. Dumortier, This conference, A 44 (2009). 